\title{
Infantile hemangioendothelioma
}

\author{
Bafana Elliot Hlatshwayo, Daba Ipfi, Sipho Sithole
}

\begin{abstract}
Introduction: Hepatic tumors in children are relatively uncommon and account for $2-3 \%$ of all pediatric tumors, however infantile hemagioendothelioma is the third most common hepatic tumor accounting for $12 \%$ of all childhood tumors. Case Report: We report the case of 12 week and 11 day old female patient who was retroviral disease (RVD) positive. The presenting complaint was abdominal distention which started soon after birth (in the first month) and rapidly increased in size. Ultrasonography showed hepatomegaly with diffuse discrete round hypoechoic lesions involving all the segments of the liver. Color Doppler demonstrated increased vascularity at the periphery of these lesions. These lesions ranged from 1-3 cm in greatest diamesion. Non-enhanced computerized tomography scan showed multiple hypoattenuating nodules, which enhanced peripherally, post-contrast, during arterial phase and showed progressive centripetal filling during porto-venous phase. Magnetic resonance imaging showed multiple round lesions of different sizes involving the whole liver parenchyma. The lesions showed low signal intensity on $\mathrm{T} 1$ weighted images and high signal intensity on T2 weighted images. The lesions did not suppress on a fat suppressing sequence. Liver biopsy was not done due to
\end{abstract}

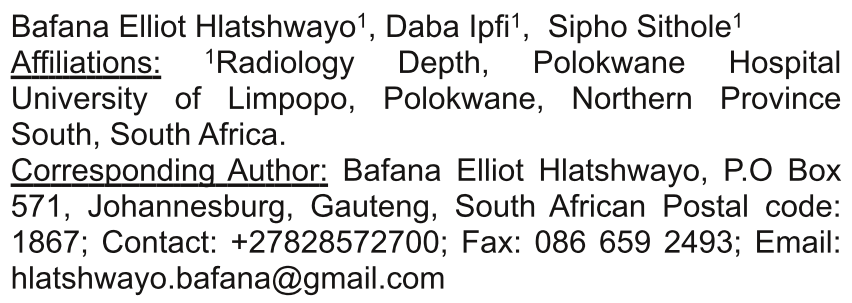

Received: 03 October 2012

Accepted: 09 October 2012

Published: 24 November 2012 highly vascular nature of the lesion and low platelet count. Conclusion: Infantile hepatic hemangioendothelioma shows some highly suggestive imaging features on both $\mathrm{CT}$ scan and MRI. Treatment depends on the presenting symptoms.

Keywords: Infantile hemangioendothelioma, Stroma, Flow void, MRI

$$
* * * * * * * * *
$$

Hlatshwayo BE, Daba I, Sithole S. Infatile hemangioendothelioma. Case Reports International 2012;1:1-4.

Article ID: 100001CRINTBH2O12

$$
* * * * * * * * *
$$

doi:10.5348/crint-2012-1-CR-1

\section{INTRODUCTION}

Infantile hemangioendothelioma is a rare benign vascular tumor arising from mesenchymal tissue and is common in lateral segment II and III of the liver. This tumor is the third most common liver tumor in children, and accounts for $12 \%$ of all childhood tumors. Other begnin pediatric liver tumors include mesenchymal harmatoma, focal nodular hyperplasia and hepatic adenoma. Malignant lesions include hepatolastoma, metastasis from neuroblastoma and lymphoma [1].

Infantile hemangioendothelioma is the most common symptomatic tumor during the first six months of life. About $70 \%$ these tumors involute spontaneously and regress within the first $12-18$ months of life but $30 \%$ need urgent management. Some tumors have life threatening complications and the management depends on the presenting symptoms. If the patient is 
asymptomatic, then conservative treatment may be adequate. Corticosteroid may be used to accelerate regression; embolization and liver transplant are other treatment options.

\section{CASE REPORT}

A 12 week and 11 day old female patient presented with complains of abdominal distention which started soon after birth and increased rapidly in size. There was gradual failure to thrive. The patient was retroviral disease (RVD) positive. She was born by normal vaginal delivery and had uneventful birth history. Birth weight was $3.02 \mathrm{~kg}$, length was $48 \mathrm{~cm}$ and Apgar score was 10/10 at 1 and 5 minutes. At presentation the weight was $6 \mathrm{~kg}$. On physical examination abdominal circumference measured $54 \mathrm{~cm}$, and abdominal veins were visible. The liver measured $18 \mathrm{~cm}$ below the last rib and the spleen was $6 \mathrm{~cm}$ in maximum length. Laboratory investigations showed hemoglobin $8.3 \mathrm{~g} / \mathrm{dl}$ (anemia), white blood cells $1.72 \times 10^{3} / \mathrm{mm}^{3}$, platelet count $0.59 \times 10^{6} / \mu \mathrm{l}$ (low), C-reactive protein $45 \mathrm{mg} / \mathrm{dl}$, total bilirubin $46 \mathrm{~mol} / \mathrm{L}$, conjugated bilirubin $25 \mathrm{~mol} / \mathrm{L}$, alkaline phosphate $44 \mathrm{U} / \mathrm{L}$, gamma-glutamyl transferase $1425 \mathrm{U} / \mathrm{L}$. Abdominal ultrasonography showed hepatomegaly with diffused discreete round hypoechoic lesions involving all the segments of the liver. The largest lesion measured $3.7 \times 2.8 \mathrm{~cm}$. The portal and hepatic veins were not visualized. The Intrahepatic bile ducts were grossly normal. The right kidney was displaced inferiorly. The left kidney and the spleen were normal. Bowel loops were displaced inferiorly to the left. Colour doppler demonstrated increased vascularity at the periphery of these lesions. Non-enhance computerized tomography (CT) scan showed multiple hypoattenuating nodules, which enhanced periphery post-contrast during arterial phase (Figure 1A) with progressive centripetal filling during porto venous phase (Figure 1B). Magnetic resonance imaging showed multiple, rounded lesions of different sizes involving the whole liver parenchyma. The lesion showed low signal intensity on T1 weighted images (Figure 2A) and high signal intensity on T2 weighted
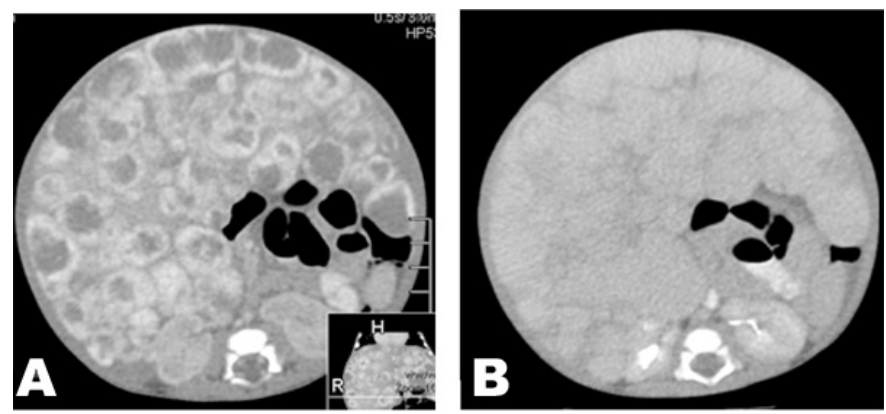

Figure 1: A) Axial CT scan post-contrast arterial phase at the level of the kidneys showed diffused hepatic masses with intense peripheral enhancement and central non-enhancing areas, B) Venous phase shows diffused enhancement of the lobulated liver segments. images (Figure 2B); the lesion was not suppressed on a fat suppressing sequences (Figure $3 \mathrm{~A}$ ). The celiac trunk and common hepatic artery were encased by the mass. Post gadolinium MRI showed enhancements of the
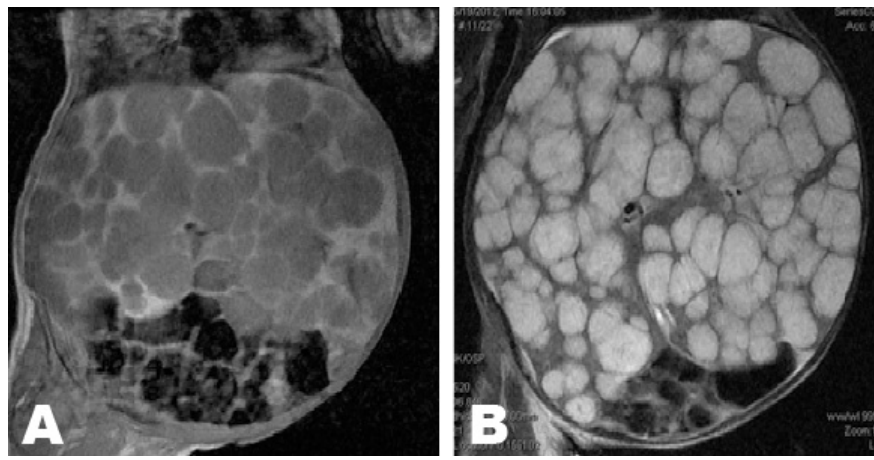

Figure 2: A) Axial unenhanced T1 weighted MRI image shows diffused nodules with low signal intensity, B) Coronal T2 weighted MRI image shows diffuse high signal intensity nodules occupying almost the entire abdomen.
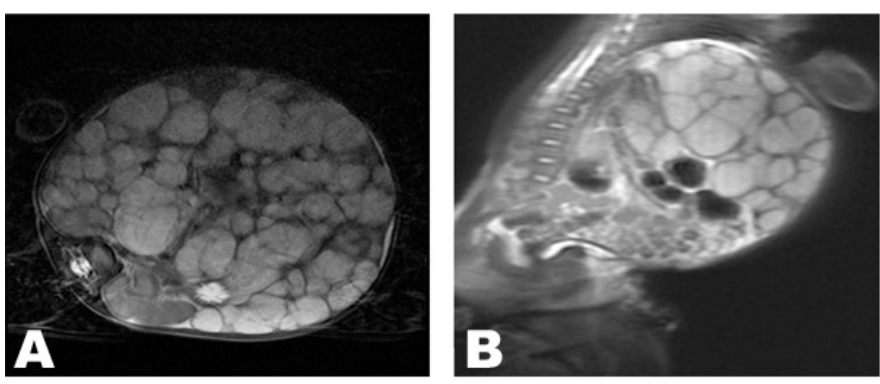

Figure 3: A) Axial MRI fat suppression shows diffused high signal nodules, B) Saggital MRI post gadolinium shows enhancement of the nodules

lesion on T1WI (T1 weighted imaging) (Figure 3B). The radiologival diagnosis was of an infantile hemangioendothelioma.

Liver biopsy was not done due to the patient's clinical condition and high vasculairty of the lesions. Patient was transfused with $60 \mathrm{ml}$ whole blood over six hours. The patient went into congestive cardiac failure while in the hospital. The patient was treated by laxis 5 mg p.o daily, propanolol $5 \mathrm{mg}$ p.o daily, neverapine $1 \mathrm{ml}$ p.o. daily, thyroxin $80 \mu \mathrm{gm}$ p.o. daily and digoxin $1 \mathrm{ml}$ orally per day. The patient expired due to complications of heart failure.

\section{DISCUSSION}

Hepatic tumors in children are relatively uncommon and account for $2-3 \%$ of all pediatric tumors. Infantile hemangioendothelioma is the $3^{\text {rd }}$ most common hepatic tumor accounting for $12 \%$ of all childhood tumors and $30 \%$ of benign liver lesions [1]. Other benign hepatic tumors include focal nodular hyperplasia (28\%), mesenchyma harmatoma (3\%) and hepatic adenoma (2\%). Hepatoblastoma is the most common malignant 
tumor and accounts for $50-70 \%$ of pediatric tumors followed by metastasis from nueroblastoma $[1,7]$.

More than $85 \%$ of the infants with infantile hemangioendothelioma present during the first six months of life $[1,2]$. The tumor is histologically benign; however, aggressive behavior is occasionally seen as a malignant transformation to a sarcoma with metastasis observed microscopically and rarely distant metastasis [2, 5]. Infantile hepatic hemangioendothelioma is a proliferative endothelial cell neoplasm that involve the liver. It is a rare tumor that may occur as a solitary lesion or multifocal nodules ranging in size from few $\mathrm{mm}$ upto $15 \mathrm{~cm}$. The tumor was first defined as a specific entity by Weiss and Enzinger in 1982 [3-5]. Infantile hemangioendothelioma are asymptomatic, and are accidentally discovered by parents as abdominal swelling or during imaging of the abdomen. The disease usually presents with the following triad: enlarged cutaneuos hemangioma, hepatomegaly and congestive cardiac failure. If untreated the infant's chances of death are 50\% within two weeks of onset [5]. At presentation most of these patients are six months of age or younger [2, 6]. Dehner et al. subdivided this tumor histologically into type 1 and type 2 [7]. These two types of Infantile hemagioendotheioma have been identified on the basis of tumor size and vascularity $[1,5]$. In type 1 the lesion is calcified and consists of multiple vascular channels with immature endothelial cells lining and fibrous stroma separating them. In type 2 lesions there is more disorganized appearing endothelial cells lining and stoma bile ducts. For most patients, needle biopsy is discouraged due to possible uncontrollable bleeding, so ultrasonography, CT scan and MRI are used for diagnosis [3, 5]. Imaging features of infantile hemagioendothelioma on ultrasonography are diffused hypo-echoic spherical masses of different sizes. Unenhanced CT scans show well-defined mass of hypo attenuation relative to a normal liver parenchyma. Contrast enhanced CT scan shows enhancement pattern with nodular peripheral accumulation of contrast. MRI T1 weighted images show diffused nodules of low signal intensity. MRI T2 weighted images show hyper intensity with vascular flow voids.

Treatment is based on tumor size and the severity of the symptoms. Medical therapy includes steroid and interferon to accelerate the natural regression of the lesion and anti-failure therapy for patients with cardiac failure. Intervention (e.g. embolisation) is necessary if symptoms cannot be managed conservatively [8].

Surgery is recommended in stable, non-diffuse lesions. Our patient had extensive disease with normal liver tissue, so surgery was not attempted.

\section{CONCLUSION}

Diagnosis of infantile hemangioendothelioma tumor can be made by both CT and MRI scans. Most of the hemagioendotheliomas regress within 12-18 months of life. Corticosteroid or interferon is used to stop further growth of the tumor and to accelerate regressions.

$* * * * * * * * *$

\section{Acknowledgements}

We are thankful to Thobakgale Moswatse Jacob our Radiographer for retrieving and preparing images for this case study.

\section{Author Contributions}

Bafana Elliot Hlatshwayo - Substantial contributions to conception and design, Acquisition of data, Analysis and interpretation of data, Drafting the article, Revising it critically for important intellectual content, Final approval of the version to be published

Daba Ipfi - Substantial contributions to conception and design, Revising it critically for important intellectual content, Final approval of the version to be published Sipho Sithole - Analysis and interpretation of data, Revising it critically for important intellectual content, Final approval of the version to be published

\section{Guarantor}

The corresponding author is the guarantor of submission.

\section{Conflict of Interest}

Authors declare no conflict of interest.

\section{Copyright}

(C) Bafana Elliot Hlatshwayo et al. 2012; This article is distributed under the terms of Creative Commons Attribution 3.0 License which permits unrestricted use, distribution and reproduction in any means provided the original authors and original publisher are properly credited. (Please see www.casereportsinternational.com /copyright-policy.php for more information.)

\section{REFERENCES}

1. Roos JE, Pfiffner R, Stallmach T, Stuckmann G, Marincek B, Willi U. Infantile haemangioendothelioma. Radiographic 2003;23(6):1649-55.

2. Dachman AH, Lichtenstein JE, Friedman AC, Hartman DS. Infantile hemangioendothelioma of the liver: a radiologic-pathologic-clinical correlation. AJR Am J Roentgenol 1983;140(6):1091-6.

3. Kassarjian A, Zurakowski D, Dubois J, Paltiel HJ, Fishman SJ, Burrows PE. Infantile hepatic hemagioendothelioma: Clinical and Imaging findings and their correlation with therapy. AJR Am J Roentgenol 2004;182:(3)785-95.

4. Crocker DW, Cleland RS. Infantile hemangioendothelioma of the liver; report of three cases. Pediatrics 1957;19(4 Part 1):596-606.

5. Park EA, Seo JW, Lee SW, Choi HY, Lee SJ. Infantile hemangioendothelioma treated with high dose methylprednisolone pulse therapy. J Korean Med Sci 2001;16(1):127-9. 
6. Keslar PJ, Buck JL, Selby DM. From the archives of the AFIP. Infantile hemangioendothelioma of the liver revisited. Radiographics 1993;13(3):657-70.

7. Dehner LP, Ishak KG. Vascular tumors of the liver in infants and children. A study of 30 cases and review of the literature. Arch Pathd 1971;92:101-11.

8. Kochin IN, Miloh TA, Arnon R, Iyer KR, Suchy FJ, Kerkar N. Benign liver masses and lesions in children: 53 cases over 12 years. Isr Med Assoc J 2011;13(9):542-7. 